\title{
Urgences
}

\section{Études de cadences (extraits)}

\section{Huguette Légaré}

Numéro 4, 2e trimestre 1982

URI : https://id.erudit.org/iderudit/025054ar

DOI : https://doi.org/10.7202/025054ar

Aller au sommaire du numéro

Éditeur(s)

Urgences

ISSN

0226-9554 (imprimé)

1927-3924 (numérique)

Découvrir la revue

Citer ce document

Légaré, H. (1982). Études de cadences (extraits). Urgences, (4), 7-18.

https://doi.org/10.7202/025054ar

Ce document est protégé par la loi sur le droit d'auteur. L’utilisation des services d'Érudit (y compris la reproduction) est assujettie à sa politique d'utilisation que vous pouvez consulter en ligne.

https://apropos.erudit.org/fr/usagers/politique-dutilisation/
Cet article est diffusé et préservé par Érudit.

Érudit est un consortium interuniversitaire sans but lucratif composé de l'Université de Montréal, l'Université Laval et l'Université du Québec à Montréal. Il a pour mission la promotion et la valorisation de la recherche. https://www.erudit.org/fr/ 


\section{HUGUETTE LÉGARÉ \\ Études de cadences \\ (Extraits)}




\section{À LA VUE DE NIDS PLEINS}

les mères canards ont eu leurs oeufs la nuit dernière. j'ai pensé qu'elles avaient fort souffert. mais, voilà, je n'en sais rien, et même qu'il se peut qu'elles n'aient senti aucun mal pendant ce temps, les chevaux dormaient sous la grande tente de la campagne; et moi je rêvais que leurs nasaux constataient à distance que la marée baissait, laissant plus de terre aux oiseaux.

les chevaux savent-ils, eux, si les mères canards ont eu des douleurs en pondant leurs oeufs 


\section{PETIT MATIN LITTORAL}

la mer est cormoran. tout ce qu'il y a, un oiseau noir la mer a fait la noce, et voici un petit matin littoral la mer s'est bien vautrée, mais où sont les mêmes coeurs le lendemain

immobile au bout de la jetée, le coeur de mon cheval regarde l'eau 


\section{JE SUIS VERTE}

I'haleine des algues m'embrasse et dore ma chaleur blanche je suis verte sous le vent iodé des jupons bleus de la mer roulant sur les pierres

le déferlement des vagues $\mathrm{m}^{\prime}$ abrite comme s'il avait apporté tous les caps du monde à mes pieds pour que j'entende l'érosion-cisèlement

les algues ont des oreilles partout et montrent du doigt les cassures des falaises, sèches, odorantes, qui sentent bon les strates lavées et usées

je baigne dans des bouffées d'algues, et l'abîme rempli de couleurs réfléchies dévore le soleil entouré de galets où les sons me mouillent de mouvements poreux

I'air se pose sur moi comme si le déferlement avait demandé qu'on me fasse voir un mariage d'oiseaux autour de mon cheval qui a chaud 


\section{LA LIBERTÉ COURT AUTOUR DE NOUS}

nous sommes des cages à pigeons et les pigeons de nos cages. nous craignons parfois de périr dans le vent. nous regardons la mer comme si nous étions des poissons habitués à l'air. nous sommes des poissons excrémentiels, des rebuts de course marine. nous avons les paumes trop sèches et mal à l'aise lorsque l'air a des heures oisives. nous sommes des herbes à l'âme écailleuse. et pour aller au milieu de l'air, nous avons des amis capitaines de coquillages cassés. mais là, nos chevaux, comme des voilières ouvertes, regardent nos cages à pigeons et nous, pigeons dans nos cages 


\section{MÉNAGE Ȧ TROIS}

le cheval a la peau paisible, I'homme a la peau abstraite d'un sillon roux, et la femme compte sur ses doigts les roses peintes sur les herbes

le cheval est un oiseau qui aime, la femme une forêt blanche, et l'homme un havre sans luxe. mais les moutons, avec leur tête de chemin de terre, craignent les ménages à trois et préfèrent être vingt.

I'homme est une brise, la femme est un homme aimé, et le cheval est un homme qui fend des arbres. cependant, le taureau, avec sa tête de chemin de terre, craint les ménages à trois et préfère être seul

la femme pense à des paysages bleus parfaits, l'homme voit des chemins de terre imparfaits, et le cheval, entre sa langue de taureau et ses clôtures à moutons, travaille pour I'homme qui aime les heures qui passent, et galope pour la femme qui elle aussi aime les heures qui passent, comme si ce point commun ne craignait pas les ménages à trois.

I'homme est un gros pétiole, le cheval un liseron géant, et la femme porte des chemises vertes en remerciant les fleurs dans l'esprit avec ses bras doux. toutefois, les moutons et le taureau, le long du chemin de terre qui mène aux montagnes bleues, continuent de craindre les ménages à trois 


\section{LE VILLAGE ET LA PEUR DES ULTRASONS}

les papillons craignent les hirondelles et les villageois redoutent les ultrasons

était-il nécessaire que des craintes fabriquées s'ajoutent aux craintes naturelles

les gens du village se claquemurent à l'intérieur de la mer bleue et sous le rivage de sable doux comme des plumes brunes

puisqu'ils n'ont pas de garages, ils avalent leurs barques, le soir, avant d'aller dormir

au moins, s'ils possédaient l'instinct des oiseaux, et savaient trouver des cheminées pleines de silence parfait comme des rigoles d'eau douce en bordure de l'océan

avant de sortir, ils lavent tout le sucre qu'il y a sur leur peau, de peur que les ultrasons, ces êtres ni jaunes ni bruns encombrant un monde qu'ils décolorent, ne cherchent du nectar comme les abeilles

il y a, bien sûr, le cheval auquel on donne à manger des framboises, qui est un petit belvédère de paix

mais il ne chasse pas les ultrasons, qu'il faudrait savoir identifier, comme le poisson a appris à reconnaître les couleurs des fibres synthétiques des filets

les gens du village ont la figure tellement picorée par les ultrasons qu'ils invitent les hirondelles à voler en rase-mottes contre leurs joues

et les hirondelles se prêtent à ce jeu, sachant que le spectacle de leur petit ventre rond et doux ne fait qu'affermir la bonne entente qui existe entre elles et les villageois côtiers 


\section{PLUMULES DE CHEVAL}

I'étalon cueille la jument, sous un ciel de duvet d'immortelles, pour qu'elle s'étire, pour qu'elle invente sa cueillaison et s'admire, pour qu'elle ne cache pas son souffle renversé, son souffle grisé et lissé sous un corps

sous un ciel de duvet de terre, l'étalon prend le risque de donner à la jument sa lumière première et il cherche à voir comme elle est reçue

il étend sur les yeux de la femelle son plus beau pétale, et il cherche à savoir si elle sourit sous cette douceur et sous le duvet de l'horizon. ah! ce bel étalon et son pétale des grandes occasions

la grande occasion, à pas de loup, se fond dans l'instinct qui dit que le soir sera beau pour la jument tourmentée par d'étranges capsules de pavot et par un ciel de duvet de foin

avec sa roseur qui aime la noirceur et sa noirceur qui aime la roseur, la nuit vient sur un ciel de duvet de sable, et le calme qui chante des silences de toutes sortes attend l'accouplement crépusculaire

le halo de la lune chasse la brume rose qui entoure la tête de l'étalon. et la jument reste naseaux étonnés, cuisses complètes et croupe terrienne sous les caresses raclant sa litière de duvet d'asters bleus

I'étalon aida cet autre jour à se lever. et les plumules de l'aube, moites et lumineuses, étaient tendues contre ce matin de duvet de feuilles où la jument se découvrit non sanglante 


\section{IMPOSSIBLES CORBEAUX CORNEILLES ROUX}

sous la charpente du jour noir et jaune, mon cheval m'attend sur les plateaux de vent, moi qui ne suis qu'un pauvre orage sur toute la flore, regardant la vie avec des teullages morts

ma main n'est pas une main, mais un dôme vert, introverti. et moi qui ne suis qu'un pauvre orage sur toute la flore, j'ai aussi un menton où mon coeur a des coudes de sel gris de mousse qui chantent les impossibles corbeaux corneilles roux autour d'un cheval vivant

avec mes racines muettes et mon pauvre orage sur moimême, je ne suis que des fables d'automne blanc sur toute la flore, et la blancheur de la fable n'est même pas la blancheur d'un peu de vie vécue pour parler d'automne, il faut être plus qu'un pauvre orage au milieu jaune du terrain, où toute la flore vieillit autour de mon cheval vivant

il faut être la vie tenant un bouleau jaune entre ses doigts, et des cailloux roses de source froide entre ses pattes d'oiseau de passage dans les plants de pois. il faut être d'impossibles corbeaux corneilles roux autour d'un cheval vivant 


\section{LA PIE SUR LE PERRON}

quelle est donc cette paresse qui me donne parfois envie de dormir quand mon homme aux muscles nerveux demande que nous allions voir les cabanes de la rive? il regarde mes cheveux et voudrait $\mathrm{m}^{\prime}$ emmener à cheval me faire décrire par les trapèzes d'embruns et la dérive clouée

je n'ai pas envie de sortir mes joues dans le froid, mais seulement de regarder par les carreaux la pie qui saute sur le perron

je veux surcharger ma tête d'images revues de canards ridés, d'homme et de cheval au bord des vagues

j'ai besoin de revoir l'image de mes doigts avec l'instrument qu'est le temps lent et visible et puis la chair de mes paupières tombe de fatigue, et l'homme souhaite aller loin dans les sentiers. quelle est cette paresse qui me donne envie de n'être ni lièvre ni homme à la tristesse puissante et à la joie invisible, mais seulement la pie sur le perron

je n'irai pas flotter au vent comme un arbre, ou être un estuaire rond d'érables comme une montagne

je veux avoir une journée à moi pour rêver seule avec ma pie sur le perron, faire des rêves de toutes sortes, avoir le temps de les parfaire, nettoyer ceux de l'année dernière, me faire de nouvelles faiblesses fières et des lucidités tendres

je veux remonter jusqu'à l'époque où furent inventés les violons, et avoir pour la journée un amour artiste pour ma pie sur le perron

quand mon homme veut $\mathrm{m}^{\prime}$ emmener à la mer qui monte jusque dans le petit bois, quelle est cette paresse qui me fait préférer refuser parfois? quelle est donc cette envie que j'ai de rester avec une sorte de perfection de rêves liés, où toute la réalité est à revoir, et avec une pie qui saute sur le perron 


\section{STUPÉFACTIONS}

il arrive qu'il y ait de grandes stupéfactions, et qu'on ne croie plus guère pouvoir rouler de mots

on reprend pourtant son blizzard, et l'on remarque une espèce d'oiseau qui nous est nouvelle, et l'on n'y pense pas que l'oiseau qu'on a vu est peut-être en deuil 


\section{AVIAIRE}

un bateau de papier suivait à la course, entre les algues blanches des édifices, les autobus au front carré

j'avais le fond du coeur aviaire mais non pas résistant à toutes les tristesses dans le fourmillement des moyens de transport

la ville était' rieuse et consommait sa rosée. le vent dépeçait le printemps pour trouver le double fond de mon corps, un fond de coeur aviaire mais non pas résistant à toutes les tristesses dans le fourmillement des moyens de transport

notre avenir avait de l'avenir, messieurs, si l'on considérait que nous partirions pour là où il y a des boeufs sur les marguerites, à côté de journaux jaunes que les oiseaux déchirent pour en faire des maisons aviaires et résistant à toutes les époques dans le fourmillement des moyens de transport

je me sentais panier de paille rempli de laitue sur le trottoir. je me sentais signalisation avec pots de fleurs et cordes à linge de tiges de fougère dans ces matins inadaptables, des matins aviaires, poétiques et résistant au fourmillement des moyens de transport

je suis partie pour là où sont les boeufs et les marguerites, car je ne pouvais atteindre ma pleine grandeur de plante sur un bateau de papier suivant à la course des autobus au front carré entre les algues blanches des édifices et dans le fourmillement des fantômes de chevaux de laitiers 